Supporting Information for

\title{
Directional and Reconfigurable Assembly of Metallodielectric Patchy Particles
}

\author{
Zuochen Wang, Zhisheng Wang, Jiahui Li, and Yufeng Wang* \\ Department of Chemistry, The University of Hong Kong, Pokfulam Road, Hong Kong SAR, China \\ *To whom correspondence should be addressed: wanglab@hku.hk
}

\section{Contents:}

Figure S1-S19

Supplementary Discussion 1-2

References
Page S2-S18

Page S13-S18

Page S19 


\section{SUPPLEMENTARY FIGURES.}

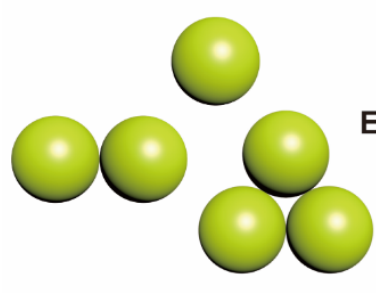

Clusters

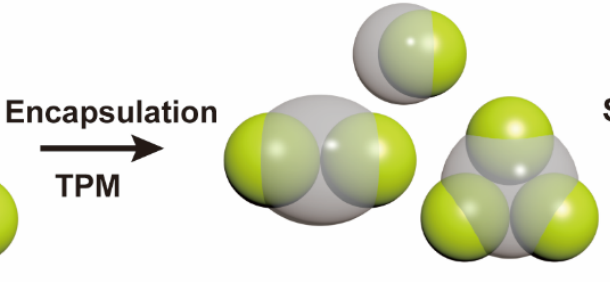

Encapsulated

clusters
Solidification

AIBN, $80^{\circ} \mathrm{C}$

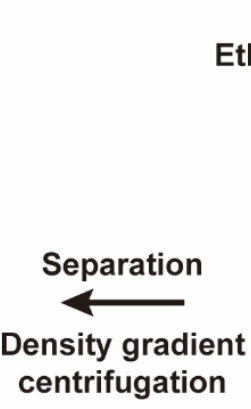

Amino two-patch

particles

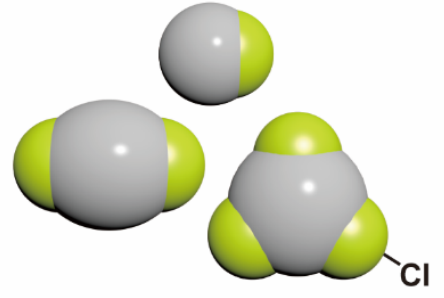

Chlorine-patch

particles

Amino-patch particles

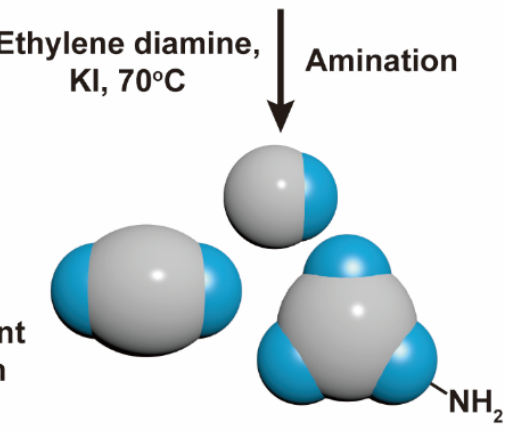

Figure S1. Schematic illustration of the synthesis of metallodielectric two-patch particles and the experimental conditions for each stage. 

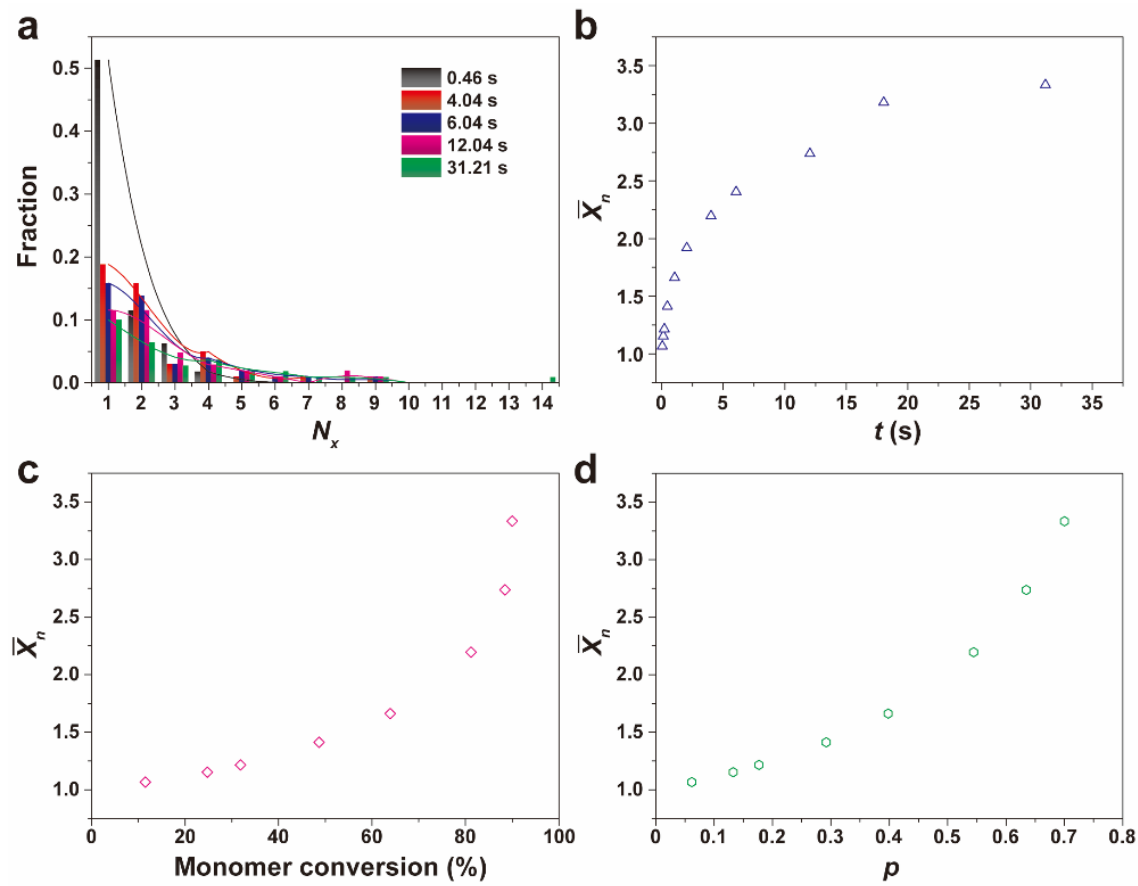

Figure S2. a, Distribution of the chain length $\left(N_{\mathrm{x}}\right)$ at different assembly times. The colloidal chains gradually become longer with the time, displaying the continuous growth. The monomer fraction decreased dramatically at the initial stage due to the formation of colloidal oligomers; the fraction of monomer then maintains even with the assembly time. b, Number-average degree of polymerization $\left(\bar{X}_{n}\right)$ varies with the assembly time. $\bar{X}_{n}=\frac{\sum n_{x} x}{\sum n_{x}}$, where $x$ is the number of patchy particles in the chain and $n_{x}$ is the number of chains containing $x$ patchy particles. The individual particles are included in the calculation. The linear relation can be observed at the initial stage, following the characteristic, reaction-controlled step-growth polymerization. With the increase of assembly time, the polymerization rate decreases as the assembly enters a "diffusion controlled" stage, because the colloidal chains are restricted in a quasi-two-dimensional plane and the kinetics are largely reduced. ${ }^{1}$ Plots showing $\bar{X}_{n}$ as a function of the c, monomer conversion and $\mathbf{d}$, extent of reaction $(p)$. Monomer conversion $=\frac{n-n_{i}}{n} \times 100 \%$, where $n$ is the total number of patchy particles and $n_{i}$ is the number of individual patchy particles. $p=\frac{n-n_{t}}{n}$, where $n_{t}$ is the number of colloidal chains after time $t$ (monomer is included). The $\bar{X}_{n}$ grows slowly when the monomer conversion is low; while the curve elevates steeply at high monomer conversion. 


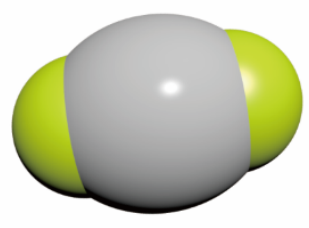

Field On
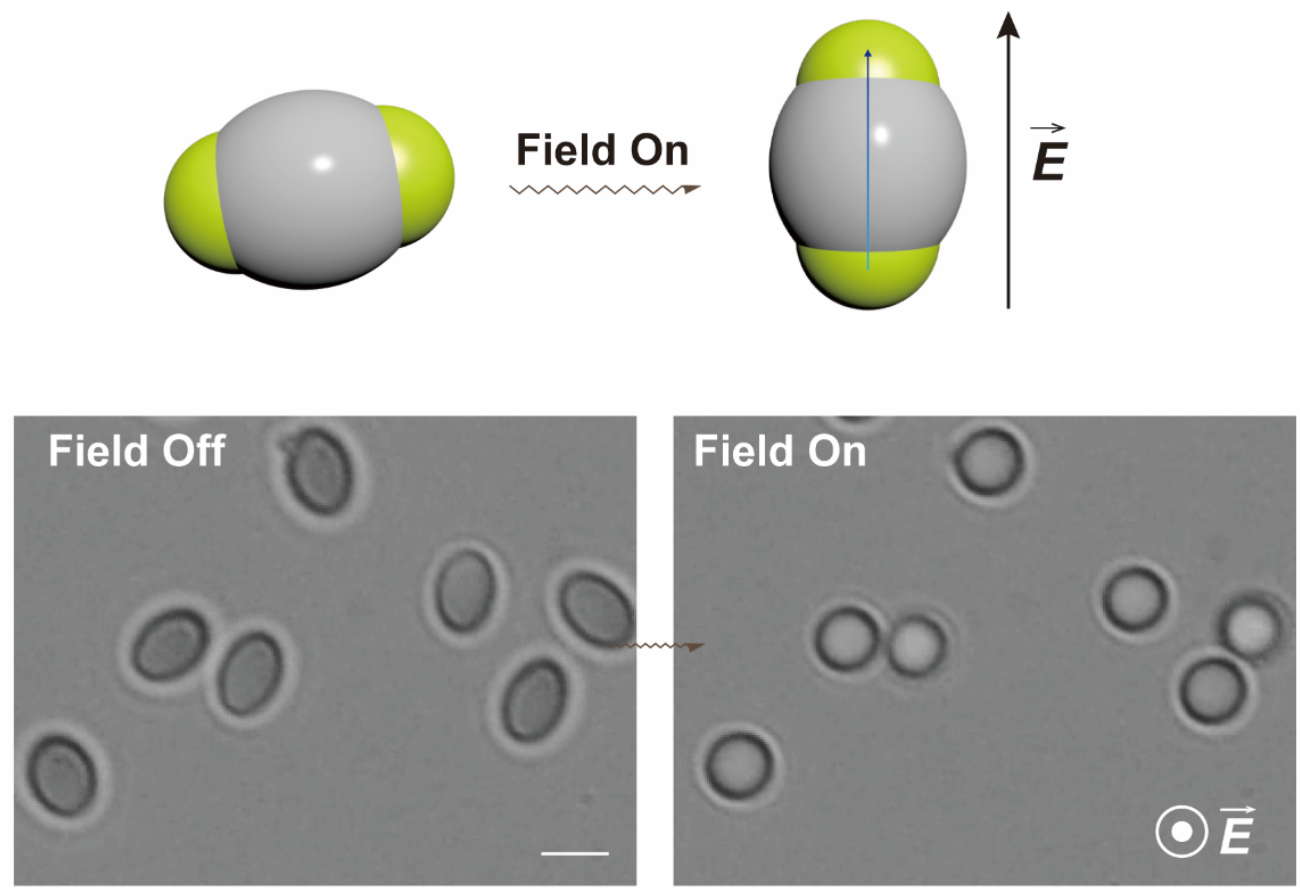

Figure S3. Dielectric ellipsoidal particles align their long axis to the field direction. Scale bar: 2 $\mu \mathrm{m}$.

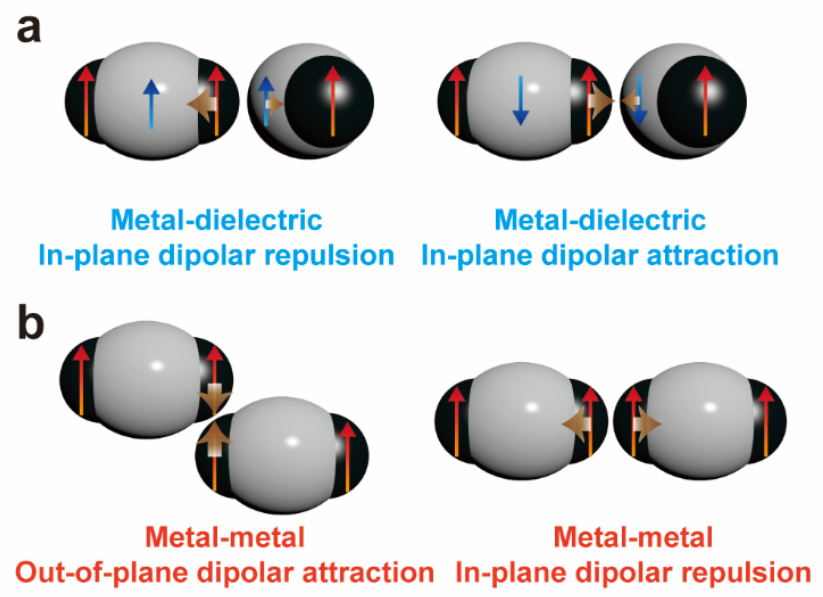

Figure S4. a, The dielectric matrix and metallic patches can display either the in-plane attraction or repulsion, depending on the sign of polarization. $\mathbf{b}$, The metallic patches are attractive with each other via out-of-plane dipolar attraction, while they are repulsive when they are in plane. 


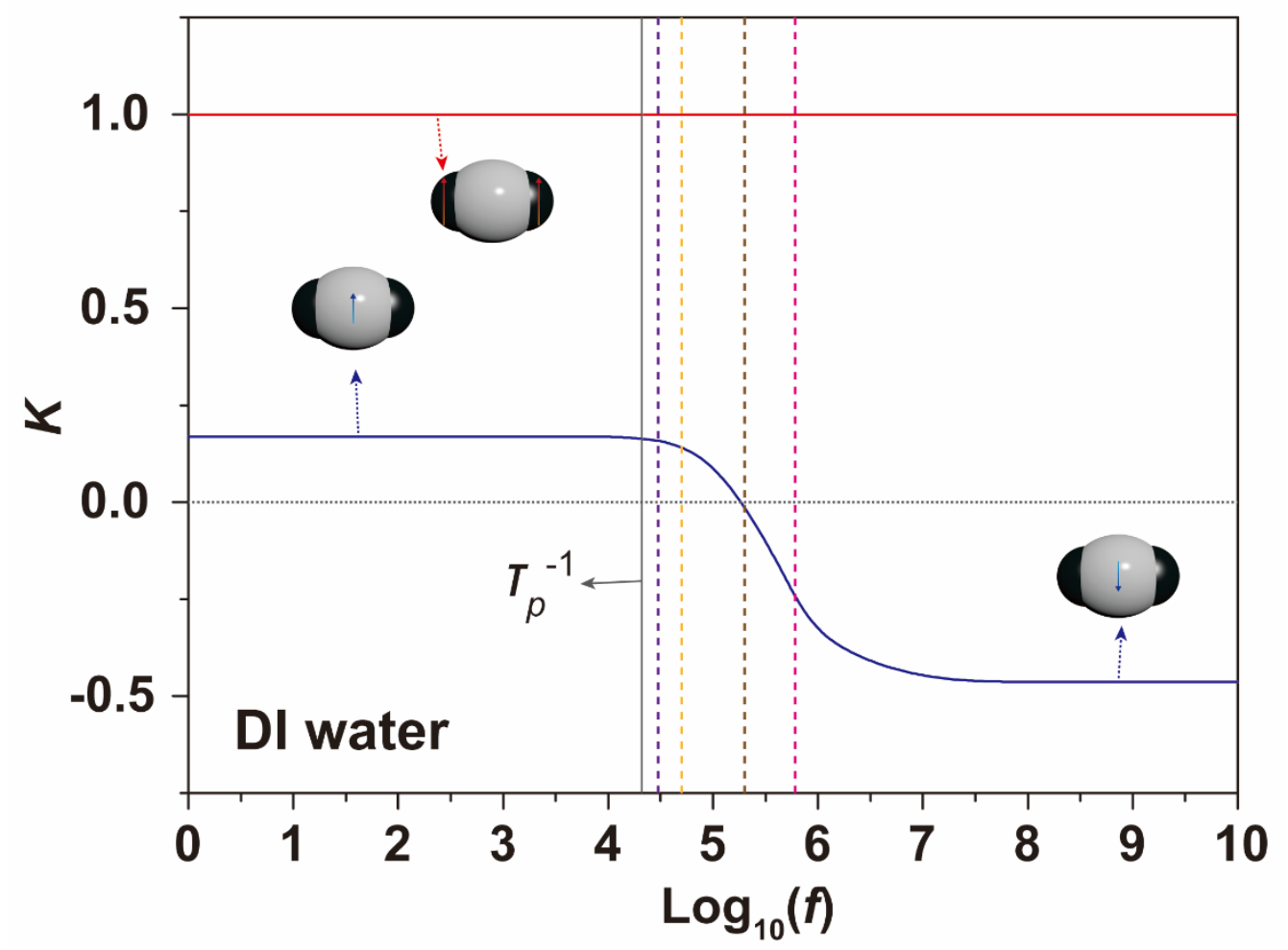

Figure S5. The calculated dipole coefficient $K$ (real part) plotted against AC frequency for metallic patches (red) and dielectric matrix (blue) in DI water. The dashed lines represent different frequencies with the same color shown in Figure 3a $(30,50,200,600 \mathrm{kHz})$.
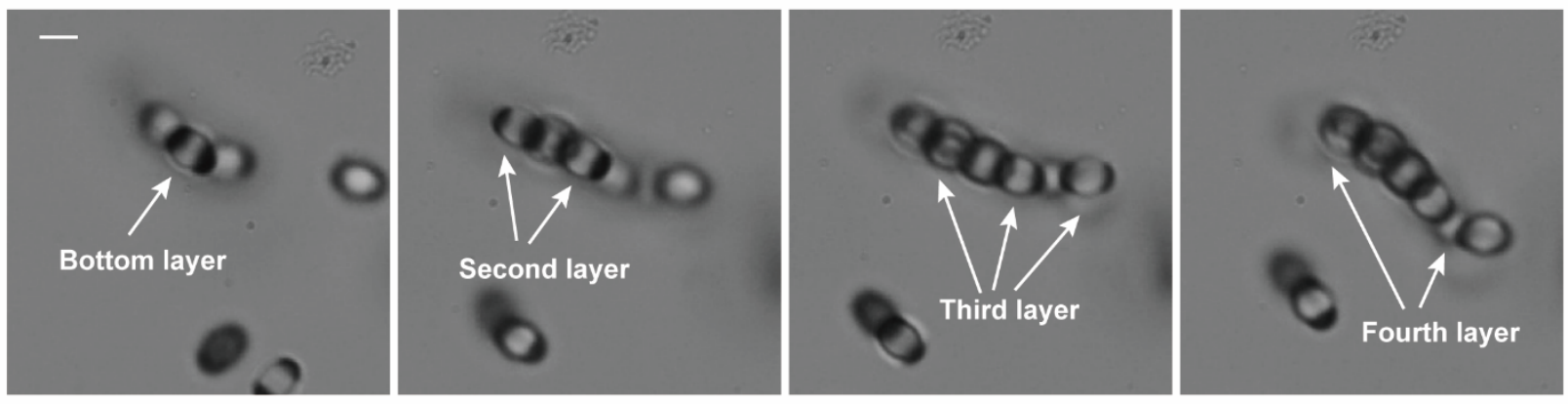

Figure S6. The assembly structure observed at different focal planes showing layers of the openbrick wall structure. Scale bar: $2 \mu \mathrm{m}$. 


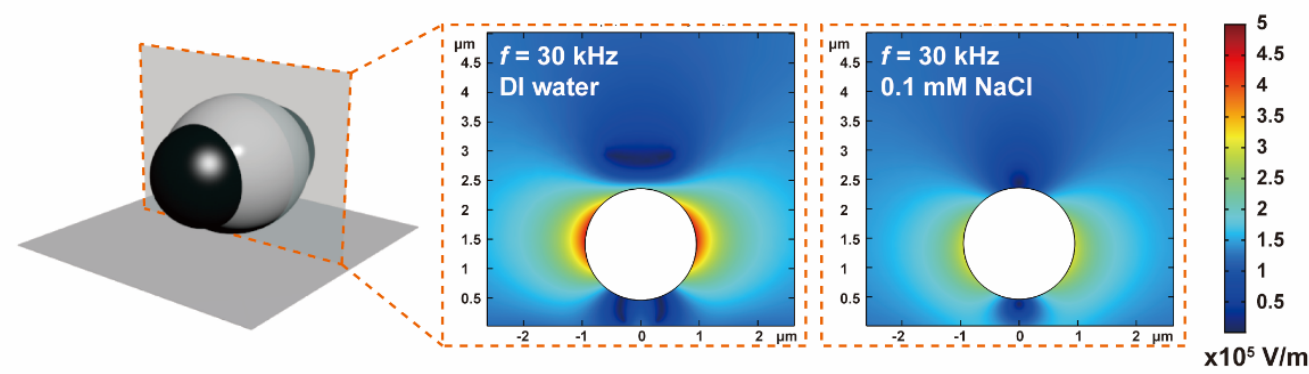

Figure S7. The numerical simulation of the local nonuniform electric field around the dielectric matrix (x-z plane) in different mediums (DI water and $0.1 \mathrm{mM} \mathrm{NaCl}$ ).

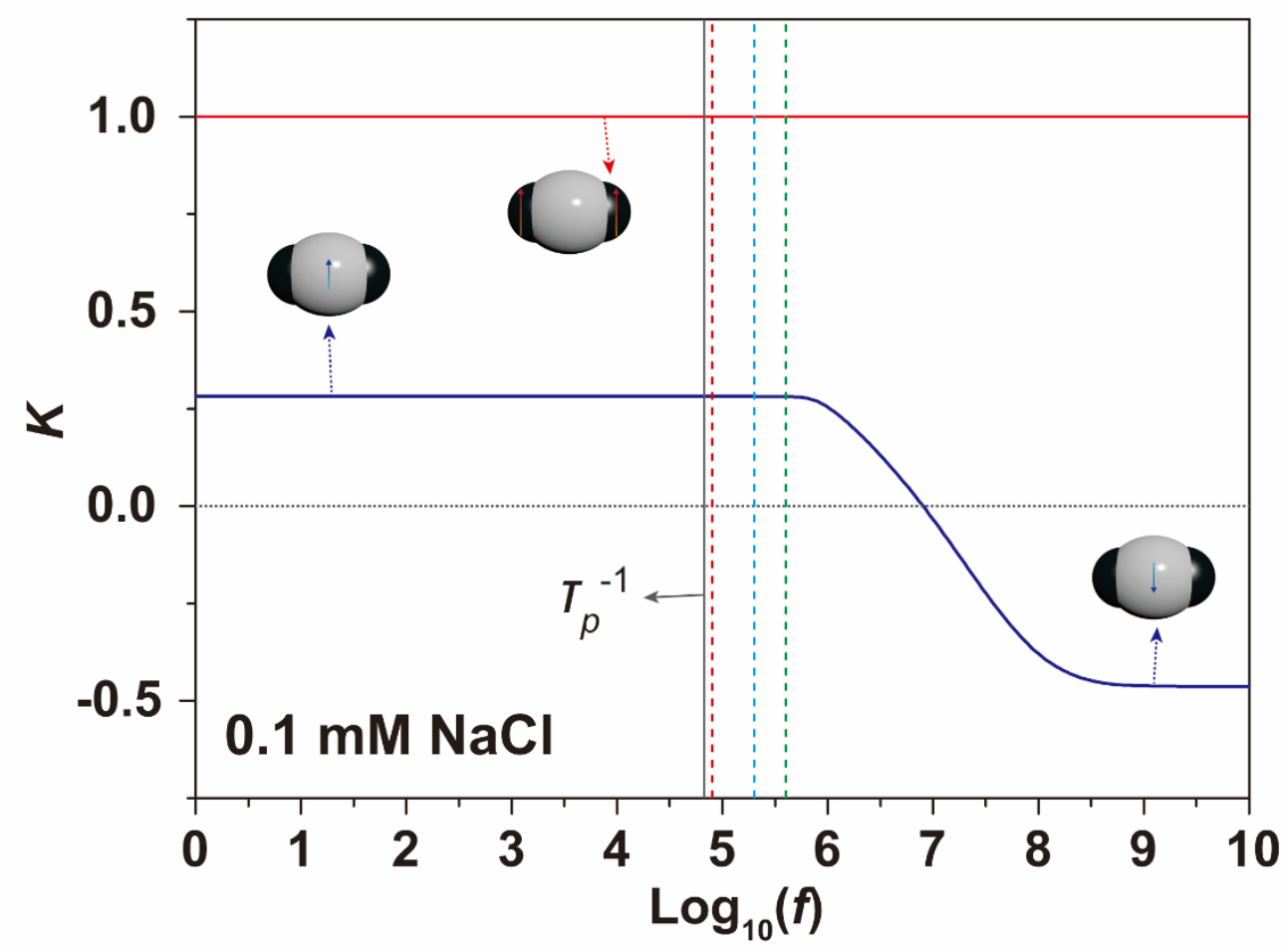

Figure S8. The real part of the dipole coefficients $K$ (calculated) plotted against frequency for metallic patches (red) and dielectric matrix (blue) in $0.1 \mathrm{mM} \mathrm{NaCl}$ aqueous medium. The dashed lines represent different frequencies with the same color shown in Figure S9 (80, 200, $400 \mathrm{kHz}$ ). 


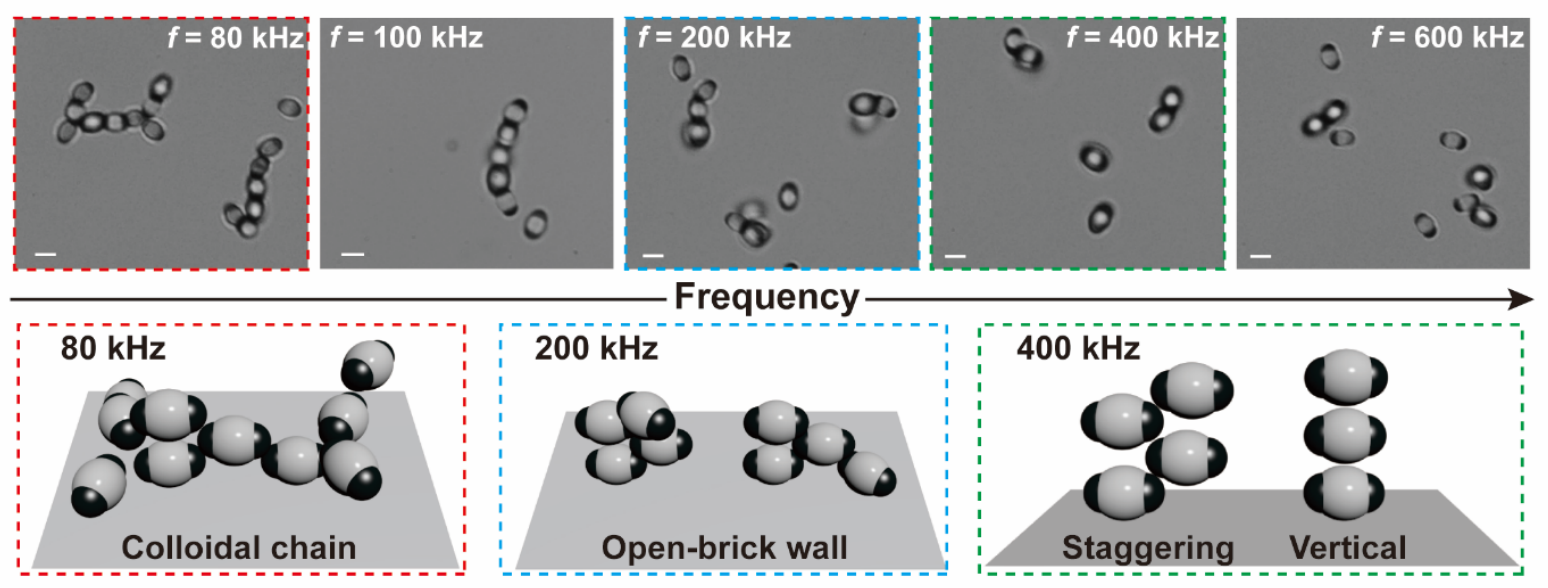

Figure S9. Frequency-dependent assembly of patchy particles in $0.1 \mathrm{mM} \mathrm{NaCl}$ solution. Scale bar: $2 \mu \mathrm{m}$.

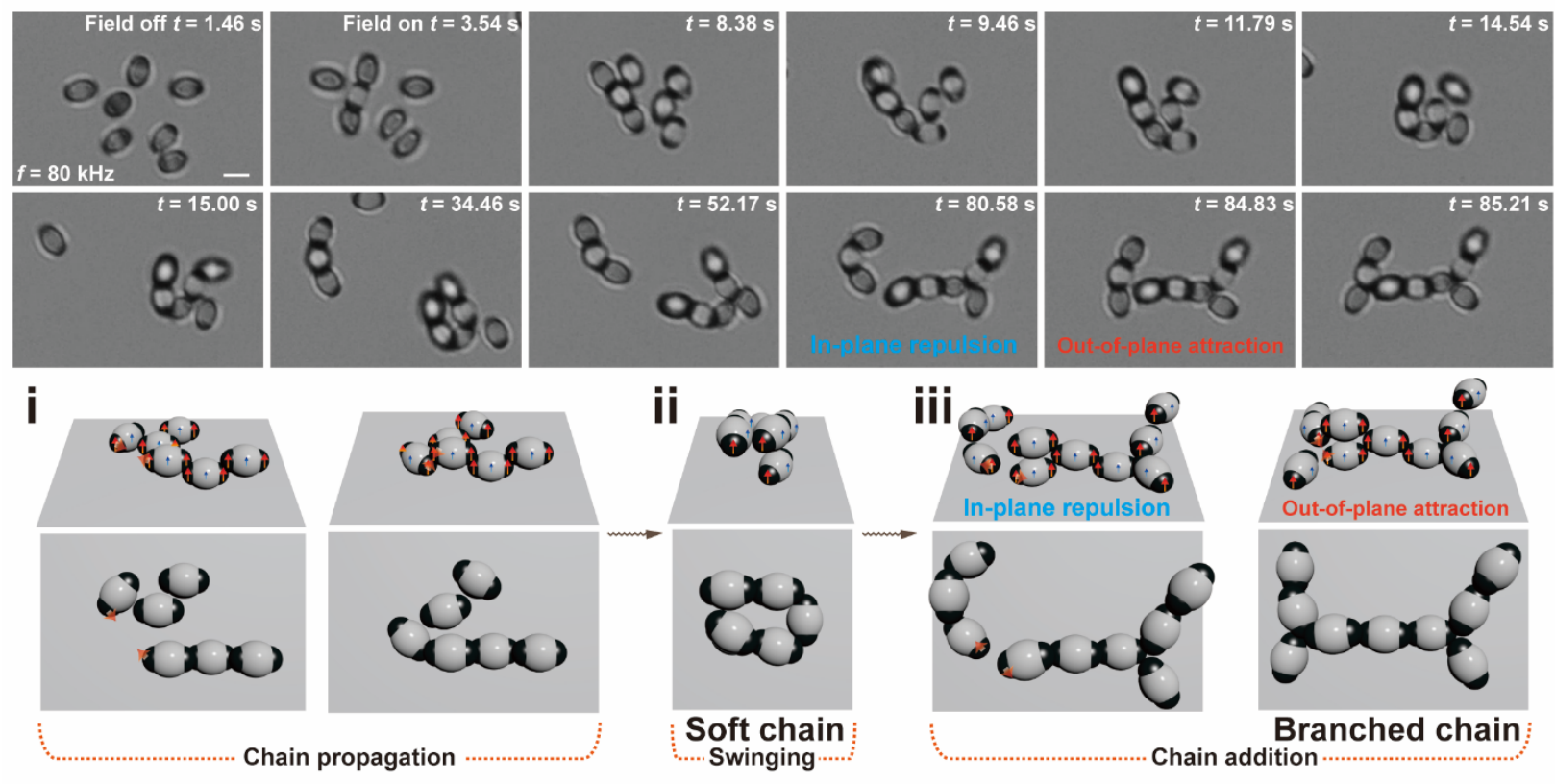

Figure S10. Time-lapse optical microscope images and cartoons of the formation process of flexible and branched chains in $0.1 \mathrm{mM} \mathrm{NaCl}$ solution, at $f=80 \mathrm{kHz}$. Scale bar: $2 \mu \mathrm{m}$. 

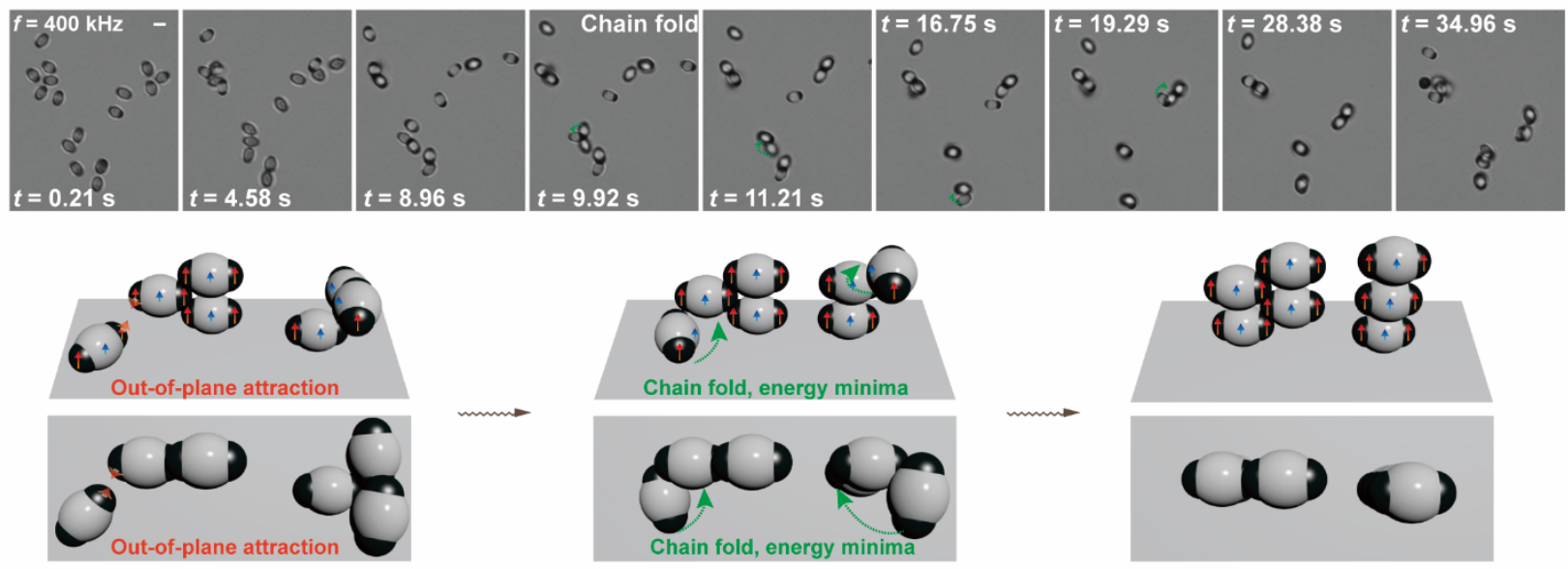

Out-of-plane attraction

Reconfiguring

Staggering stacks and vertical chains

Figure S11. Time-lapse optical microscope images and cartoons of the formation kinetics of staggering stacks and vertical chains, realized in $0.1 \mathrm{mM} \mathrm{NaCl}$ solution, at $f=400 \mathrm{kHz}$. Scale bar: $2 \mu \mathrm{m}$. 


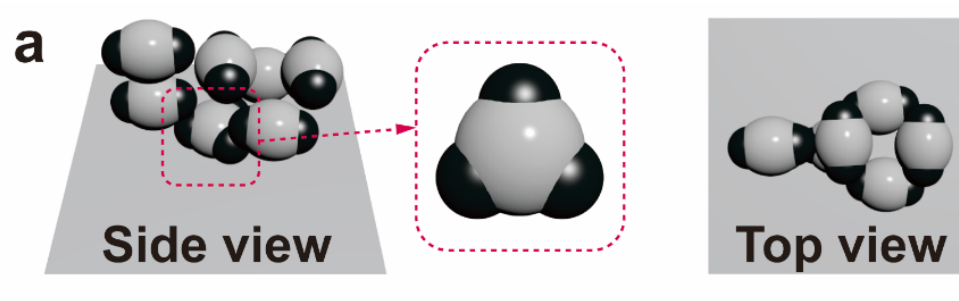

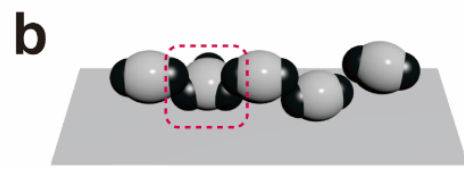

Side view

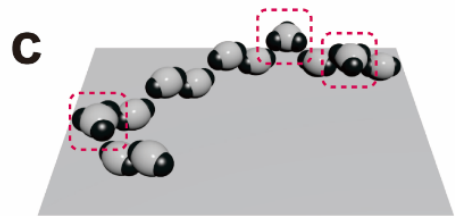

Side view

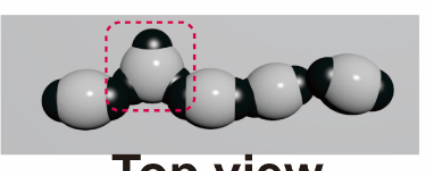

Top view

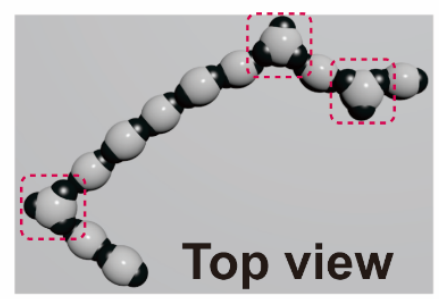

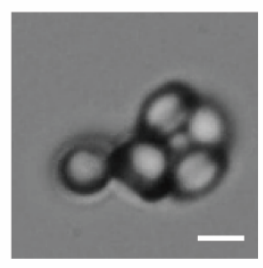
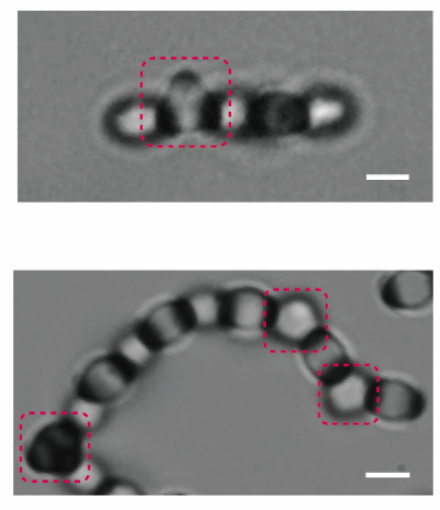

Figure S12. Various structures are obtained when a small number of three-patch particles are coassembled with two-patch particles. a, Ring structure at $f=100 \mathrm{kHz}, 0.1 \mathrm{mM} \mathrm{NaCl}$. b, Two patches of three-patch particles can be occupied by other particles in DI water at $f=30 \mathrm{kHz}$. c, Zig-zag chains can be formed when more three-patch particles join the assembly (in DI water and at $f=30$ kHz). Scale bar: $2 \mu \mathrm{m}$. 

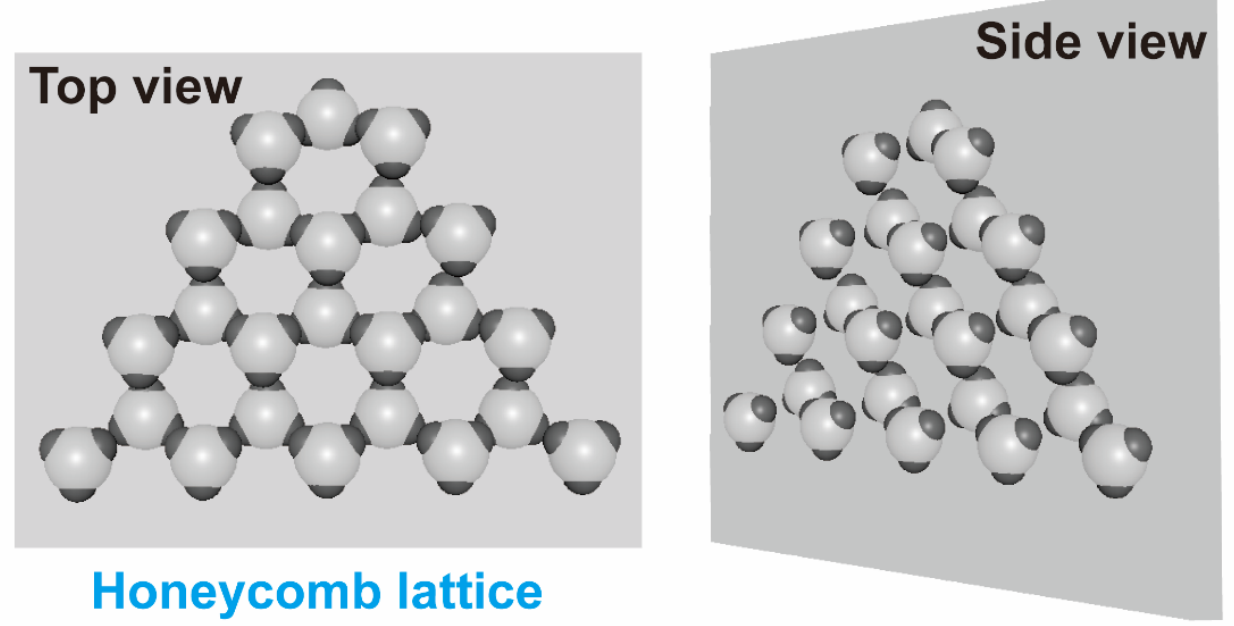

Figure S13. According to our strategy, the three-patch particles may form two-layer 2D honeycomb structures. 

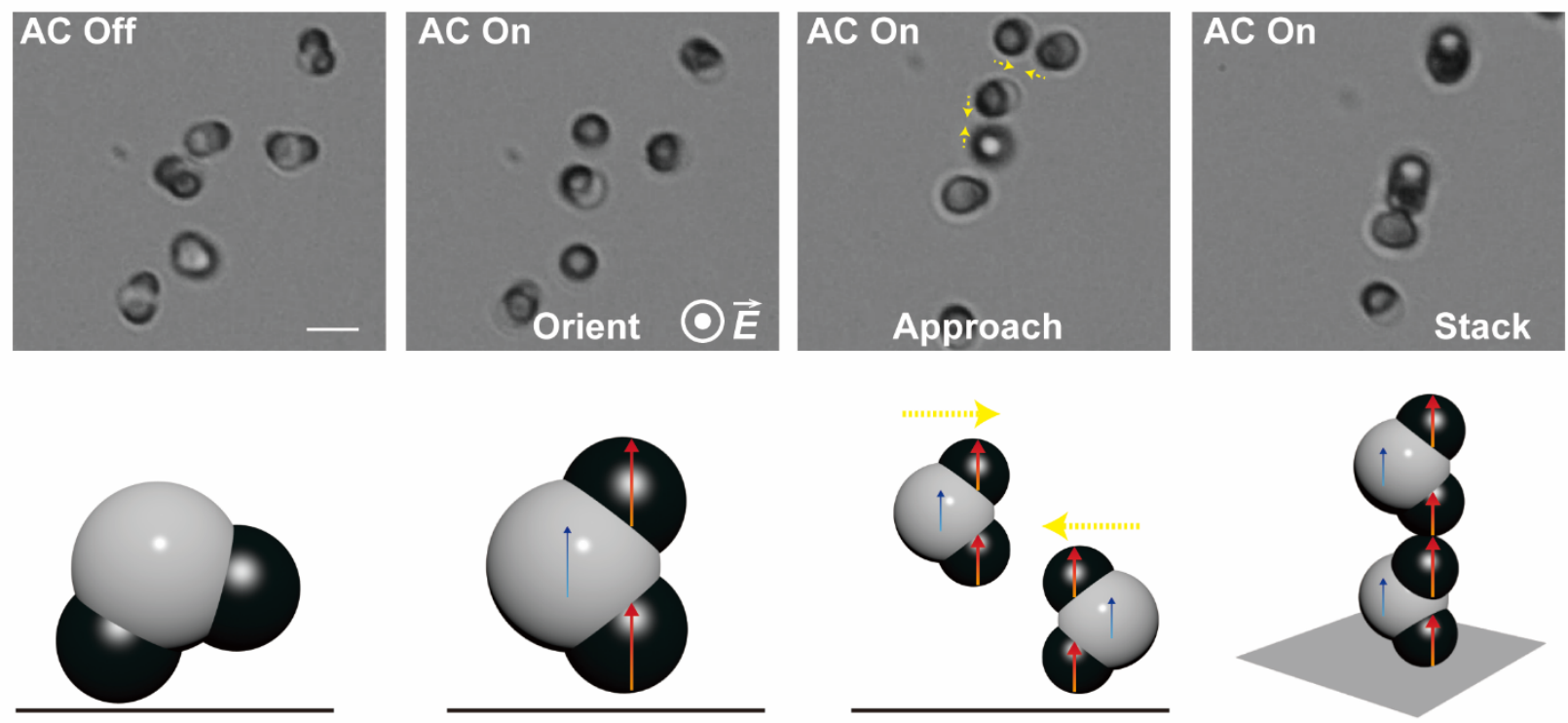

Figure S14. The orientation and assembly of bent two-patch particles under AC electric fields, realized in $0.1 \mathrm{mM} \mathrm{NaCl}$ solution at $f=100 \mathrm{kHz}$. The largest dipole moment of bent patchy particles is parallel to the line connecting the mass center of the two metallic patches. The particles therefore align their largest dipoles to the field direction showing a "stand-up" configuration at high frequency where the induced dipoles dominate. Particles with this configuration can also stack with their metallic patches. Scale bar: $2 \mu \mathrm{m}$. 
a
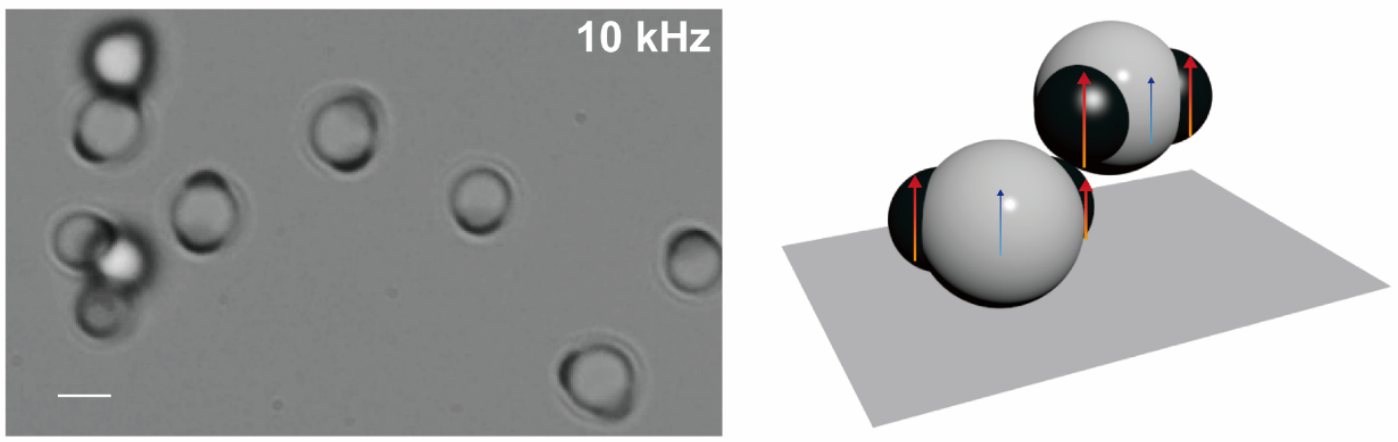

b
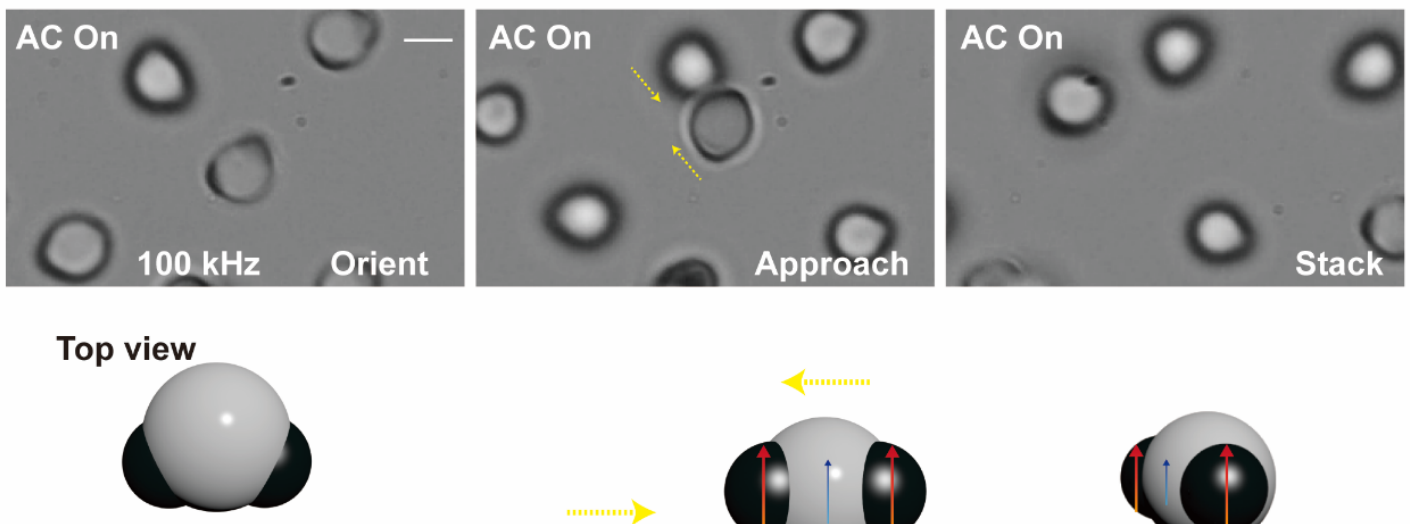

Side view
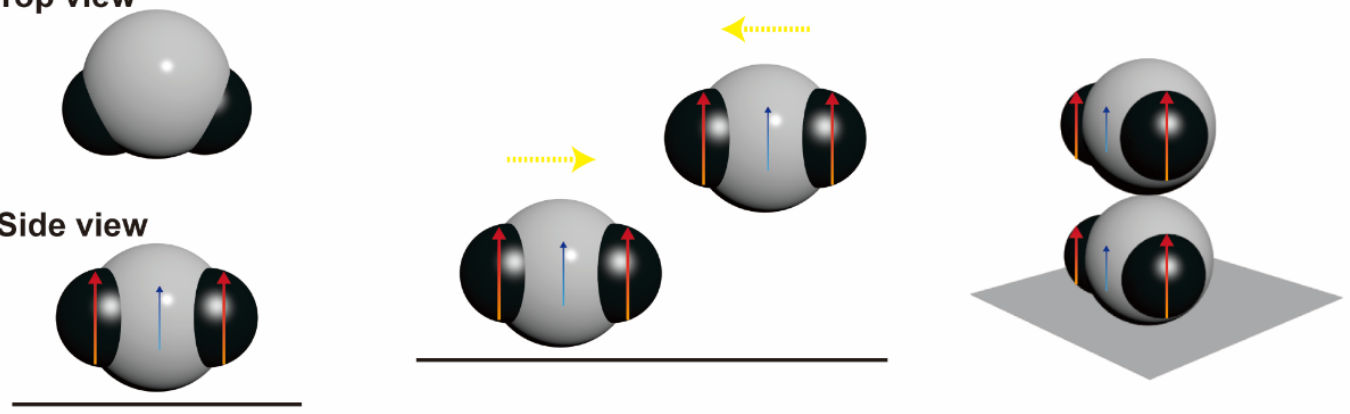

Figure S15. The orientation and assembly of two-patch particles with a smaller aspect ratio (small patch and large particle matrix). a, The directional bonding is rare and hardly extends due to the small patch size with weak dipolar attraction as well as steric hindrance from the matrix (in DI water, at $f=10 \mathrm{kHz}$ ). $\mathbf{b}$, More generally, patchy particles prefer to stack due to the weaker DEP attraction (because of smaller metallic patches) from conducting substrate (in DI water, at $f=100$ $\mathrm{kHz}$ ). Scale bar: $2 \mu \mathrm{m}$. 


\section{SUPPLEMENTARY DISSCUSION.}

\section{Parameters used for calculation.}

Debye length $\kappa^{-1}=307 \mathrm{~nm}$ for deionized water and $30 \mathrm{~nm}$ for aqueous $0.1 \mathrm{mM} \mathrm{NaCl}$ solution; temperature $T=298 \mathrm{~K}$; relative permittivity of the solvent $\varepsilon_{m}=78.5$; relative permittivity of the TPM particle $\varepsilon_{p}=3.9$; viscosity of water $0.89 \times 10^{-3} \mathrm{~Pa} \cdot \mathrm{s}$. Conductivity of DI water is $1.70 \times 10^{-4}$ $\mathrm{S} / \mathrm{m}$, where the diffusion coefficient $D^{+}=9.3 \times 10^{-9} \mathrm{~m}^{2} / \mathrm{s}$ corresponds to $\mathrm{H}^{+}$and $D^{-}=1.1 \times 10^{-9} \mathrm{~m}^{2} / \mathrm{s}$ corresponds to $\mathrm{HCO}_{3}{ }^{-}$, which are the dominant ionic species present in deionized water due to the absorption of $\mathrm{CO}_{2}$ from air. Surface conductivity of dielectric particles in DI water is $2.74 \times 10^{-4}$ $\mathrm{S} / \mathrm{m}$. For the $0.1 \mathrm{mM} \mathrm{NaCl}$ solution, the conductivity is $4.28 \times 10^{-3} \mathrm{~S} / \mathrm{m}$, and we use $D^{+}=1.3 \times 10^{-9}$ $\mathrm{m}^{2} / \mathrm{s}$ and $D^{-}=2.0 \times 10^{-9} \mathrm{~m}^{2} / \mathrm{s}$. Surface conductivity of dielectric particles in $0.1 \mathrm{mM}$ aqueous $\mathrm{NaCl}$ is $9.33 \times 10^{-3} \mathrm{~S} / \mathrm{m}$.

\section{The ellipsoidal shape of patchy particle stabilizes the formed chain structures.}

For a colloidal chain assembled by three patchy particles, we analyze the experienced dipolar forces of Particle 1 from Particle 2 (attractive) and Particle 3 (repulsive) as shown in Figure S16.

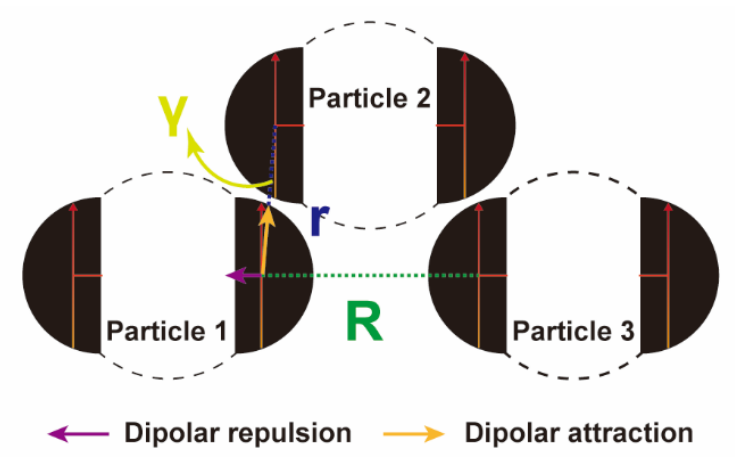

Figure S16. The experienced dipolar forces of Particle 1 from Particle 2 and 3 in a colloidal chain consisting of three patchy particles.

The dipolar force can be calculated using the equation, ${ }^{2}$ 
$F_{\text {dipole-dipole }}=\frac{3}{4} \pi \varepsilon_{0} \varepsilon_{m} a^{2} C^{2} E_{r m s}^{2}\left(\frac{2 a}{r}\right)^{4}\left[\left(3 \cos ^{2} \gamma-1\right) \hat{r}+(\sin 2 \gamma) \hat{\gamma}\right]$

where $a$ is the radius of the colloidal particles, $E_{\mathrm{rms}}$ is the root-mean-square magnitude of the applied electric field, $\varepsilon_{\mathrm{m}}$ is the relative permittivity of the medium, $\varepsilon_{0}$ is the vacuum permittivity, $r$ is the distance between the two dipoles, and $\gamma$ is the angle between the electric field and the connection of dipole centers.

For dipolar repulsion from in-plane particle 3, where $\gamma=90^{\circ}$. Therefore,

$F_{\text {repuslion }-x, 1-3}=\frac{3}{4} \pi \varepsilon_{0} \varepsilon_{m} a^{2} C^{2} E_{\text {rms }}^{2}\left(\frac{2 a}{R}\right)^{4} \cdot 1$

For the dipolar attraction from particle 2, the component in the $\mathrm{x}$-axis direction is

$F_{\text {attraction }-x, 1-2}=\frac{3}{4} \pi \varepsilon_{0} \varepsilon_{m} a^{2} C^{2} E_{r m s}^{2}\left(\frac{2 a}{r}\right)^{4}\left[\left(3 \cos ^{2} \gamma-1\right) \cdot \sin (\gamma)+(\sin 2 \gamma) \cdot \cos (\gamma)\right]$

and the dipolar attraction along the connection line is

$F_{\text {attraction }, 1-2}=\frac{3}{4} \pi \varepsilon_{0} \varepsilon_{m} a^{2} C^{2} E_{r m s}^{2}\left(\frac{2 a}{r}\right)^{4} \cdot\left(3 \cos ^{2} \gamma-1\right)(\mathrm{S} 4)$

Based on the calculational results, we obtain the following plot revealing the relation between dipolar forces and $\gamma$ (Figure S17). 


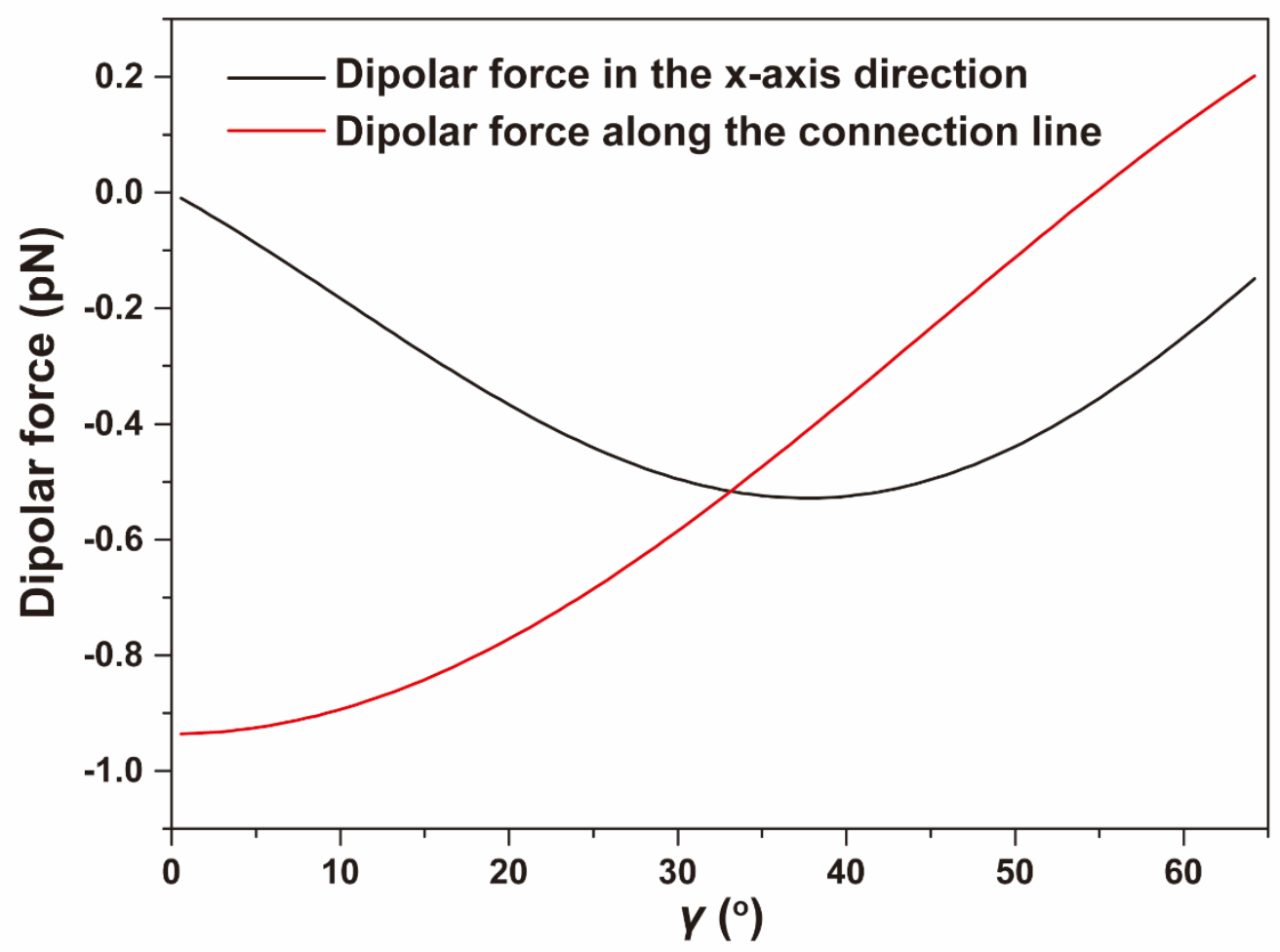

Figure S17. The calculated dipolar forces with the $\gamma$, which are either in the $\mathrm{x}$-axis direction and along the connection line.

The maximum attractive force along $\mathrm{x}$-axis is $-0.53 \mathrm{pN}$ (black line).

To form chain structure,

$F_{\text {attraction }-x, 1-2}>F_{\text {repuslion }-x, 1-3}$

Then,

$R>1.03 \cdot r$. ( $R$ refers the distance between particle 1 and $3, r$ refers to the distance between particle 1 and 2)

For example, in the system of three close-packed gold spheres, $R$ equals to $r$, the formation of the similar structure is unfavored. To fulfill the requirements of dipolar forces needed, we should try to increase the $R$ - the distance between the particle 1 and 3. One way is to lower down the height of particle 2, which increases $R$ but makes angle $\gamma$ between dipole 1 and 2 bigger. This leads to a weaker attraction force along the connection line and a decreased stability of the system according 
to Figure S17. An alternative way is to use an extended ellipsoidal particle, making use of the dielectric matrix as a spacer to reduce the in-plane dipolar repulsion. When serving as building blocks and forming a colloidal chain, Particle 1 experiences a weaker in-plane repulsive force from Particle 3, while the attractive force along the connection line can be maintained, contributing to a steady assembly and configuration.

\section{The dipolar energy of different configurations.}

For two colloidal spheres with radius $a_{A}$ and $a_{B}$ under an $\mathrm{AC}$ electric field, the dipolar interactions between two spheres can be calculated by using the equation $\mathrm{S} 5:^{3}$

$U_{A B}=\frac{4 \pi \varepsilon_{0} \varepsilon_{m} \operatorname{Re}\left(K_{A}^{*} K_{B}\right) a_{A}^{3} a_{B}^{3} E_{0}^{2}\left(1-3 \cos ^{2} \gamma\right)}{r_{A B}^{3}}$

where $K$ is the complex dipole coefficient, * indicates the complex conjugate, $E_{0}$ is amplitude of the applied field, $r_{A B}$ the distance between the two dipoles.

A variety of assembled structures emerge at different field frequencies as a result of multiple interactions exerted. The dipolar energy of dispersed particles is zero, and the particles therefore assemble to reduce the dipolar energy. To disclose the contribution of each interaction, we calculate and compare the dipolar energy of different configurations given in Figure S18. 


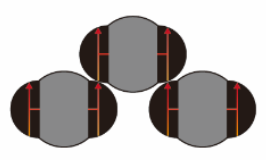

Chain
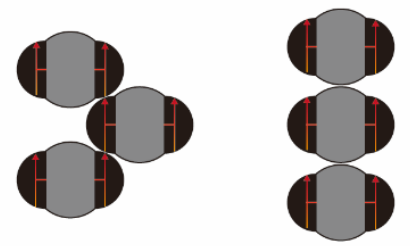

Staggering
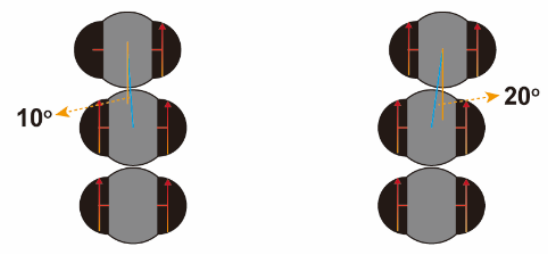

Vertical

$10^{\circ}$ migration $20^{\circ}$ migration

Figure S18. Configurations assembled by three particles used for calculating the dipolar energy.

According to our calculation of dipolar energy (Figure S19), the staggering configuration is the lowest energy state, while the vertical stacking is a local energy minimum. To verify this local energy minimum, the energy of two slightly shifted configurations are calculated where the uppermost particle shifts off from the $z$-axis by 10 degree and 20 degree respectively. The result reveals that small imposed disturbance indeed elevates the dipolar energy.

For the staggering configuration, formed at high $\mathrm{AC}$ frequencies when the EDL screening effect becomes negligible, such configurations are adopted to avoid the in-plane repulsion between two metallic patches. Yet, possible torques will be generated for the mono-bonded patchy particles in such configurations, exerted to the unbounded metallic patches by forces e.g., DEP and gravity, which may increase the energy. Some patchy particles hence form the vertical chains. Therefore, staggering configurations and vertical chains are prevailing at high frequency. 


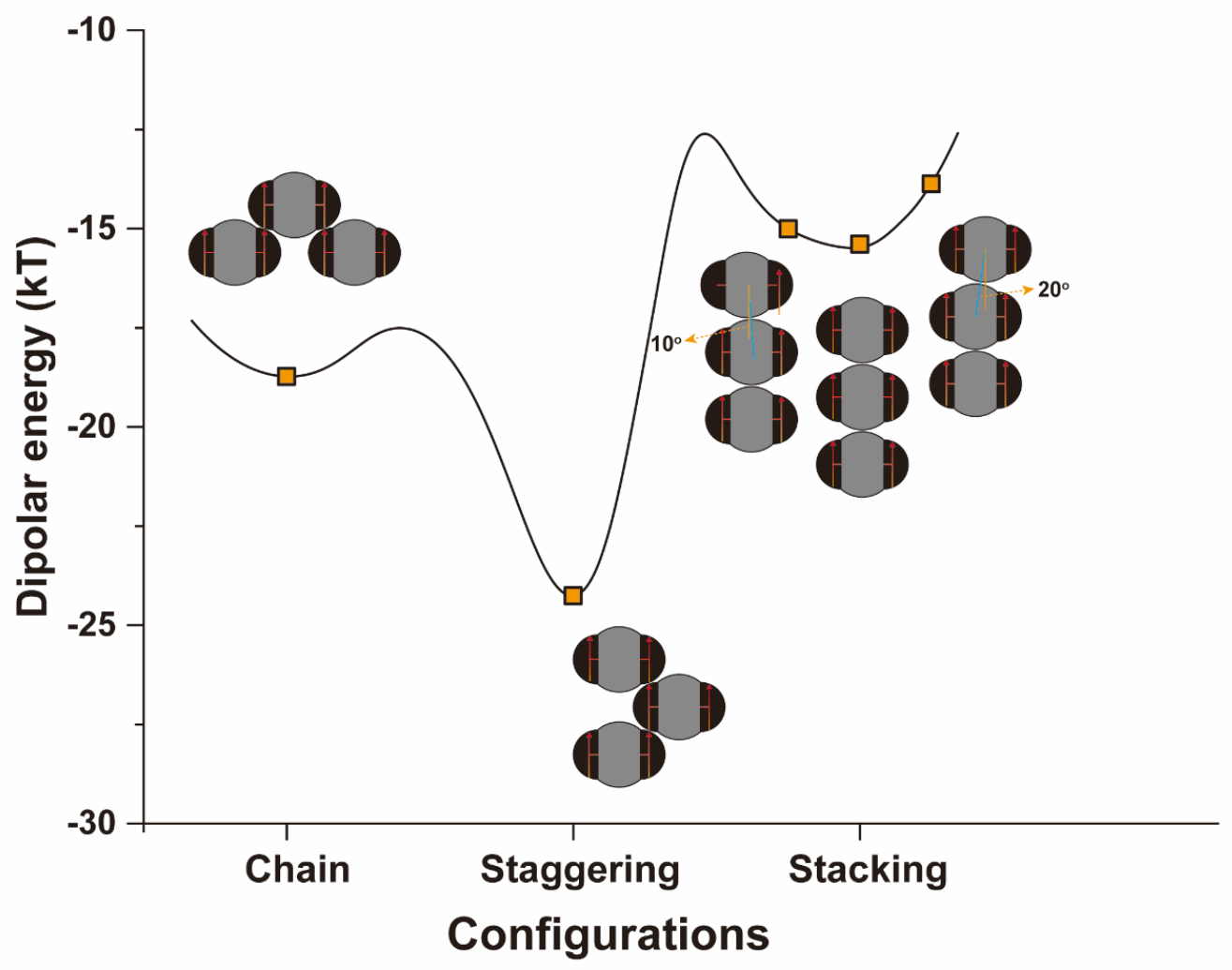

Figure S19. Dipolar energy landscape of various configurations assembled by three two-patch particles; only dipolar interactions are considered for the calculation.

Yet, apart from dipolar energy, the influence of DEP force and EDL is also non-negligible at low frequency. DEP from the conducting substrate traps the particles inhibiting stacking of the assembled structures. Meanwhile, the induced dipoles are partially screened by EDL, greatly reducing the in-plane dipolar repulsion between patchy particles. Taken together, the particles therefore form chain structures to reduce the free dipolar energies at lower frequency. 


\section{REFERENCES}

1. Li, W.; Liu, B.; Hubert, C.; Perro, A.; Duguet, E.; Ravaine, S., Self-Assembly of Colloidal Polymers from Two-Patch Silica Nanoparticles. Nano Res. 2020, 13, 3371-3376.

2. Mittal, M.; Lele, P. P.; Kaler, E. W.; Furst, E. M., Polarization and Interactions of Colloidal Particles in Ac Electric Fields. J. Chem. Phys. 2008, 129, 064513.

3. Zhang, J.; Yan, J.; Granick, S., Directed Self-Assembly Pathways of Active Colloidal Clusters. Angew. Chem. Int. Ed. 2016, 55, 5166-5169. 